\title{
Bidirectional relationships between psychological health and dermatological conditions in children
}

This article was published in the following Dove Press journal:

Psychology Research and Behavior Management

\section{Amy E Mitchell}

Parenting and Family Support Centre, School of Psychology, The University of Queensland, Brisbane, QLD, Australia
Correspondence: Amy E Mitchell Parenting and Family Support Centre, School of Psychology, 13 Upland Road, The University of Queensland,

St Lucia 4072, Queensland, Australia

Tel +6I 73346 I202

Email a.mitchell5@uq.edu.au

\begin{abstract}
Dermatological conditions are common among children. They are a frequent cause of presentation to health care services and a leading contributor to burden of disease. Evidence supports the notion that bidirectional relationships exist between children's physical and psychological health, whereby the child's dermatological condition can impact their psychological health and well-being, while, in the reverse direction, psychological factors (eg, stress) can impact the severity and course of the child's skin disease. The psychological impact of dermatological conditions in childhood needs to be taken into account during the assessment, planning, and treatment phases of management. Likewise, the potential effect of children's emotional and behavioral difficulties on management, particularly in terms of the impact on parents' ability to implement their child's treatment plan, should be considered. This literature review summarizes the current evidence for the relationships between three common chronic dermatological conditions of childhood - atopic dermatitis, psoriasis, and urticaria - and psychological adjustment and quality of life in childhood. Overall, a general paucity of research in the pediatric context combined with limitations in terms of study design, variability in operationalization of constructs, and heterogeneity in measurement of outcomes - makes it difficult to draw firm conclusions in this area. Based on the available research, implications for successful long-term management of these conditions are discussed in terms of integrating psychological and parenting support with medical management to improve adherence, reduce disease severity, and improve quality of life for children and their families.
\end{abstract}

Keywords: atopic dermatitis, child behavior, dermatology, parenting, psoriasis, urticaria

\section{Introduction}

Dermatological conditions are among the most common reasons for child presentations to family doctors in Australia, ${ }^{1}$ and rank fourth in terms of contributors to nonfatal burden of disease at the global level. ${ }^{2}$ When these conditions become chronic in nature, they are associated with significant negative effects on physical, psychological, and social quality of life for affected children and their families. ${ }^{3,4}$

An accumulation of epidemiological data suggests that complex and bidirectional relationships exist between dermatological conditions and psychological well-being for affected individuals. This has been more extensively studied in adults, with research linking the development and exacerbation of skin disease to stress, anxiety, and depression, while, in the reverse direction, psychological symptoms have been shown to contribute to a worsening of skin disease. ${ }^{5}$ Likewise, in the pediatric context, evidence supports the notion that symptoms of a number of common dermatological conditions tend to be 
exacerbated by stress, while, at the same time, children with these conditions appear to be at increased risk of developing concurrent emotional and/or behavioral difficulties at higher rates compared with unaffected children. ${ }^{6-19}$ The complex relationships between physical and psychological health in this clinical group have important implications for the management of skin conditions in childhood, and underscore the need for careful psychosocial assessment of child and family functioning to ensure that appropriate support is provided not only to the child but also to their parents and family who are ultimately responsible for the child's management.

The purpose of this article is to provide an integrated narrative review of the research literature that examines the evidence for relationships between children's psychological well-being and three common chronic dermatological conditions of childhood: atopic dermatitis (AD), psoriasis, and urticaria. Implications for management and methodological limitations of the current research are discussed.

\section{Relationships between dermatological conditions and children's mental health} Atopic dermatitis

AD (synonymous with "atopic eczema") is an inflammatory skin condition, typically with a chronic or chronically relapsing course. Signs and symptoms include dry skin, intense pruritus (itch), and a papular rash with characteristic patterns of distribution that vary depending on the child's age. It is estimated to affect $17.5 \%$ of children globally, and around $1 \%$ of children have severe AD. ${ }^{20}$ There is a strong link between family history of atopic disease and the development of AD in children, who frequently present with $\mathrm{AD}$ first before going on to also develop asthma and/or allergic rhinitis. ${ }^{21}$ In all, around $60 \%-70 \%$ of cases develop AD by age $1^{22}$ and $70 \%-95 \%$ by age $5 .{ }^{23}$ Thus, the burden of this condition in childhood is substantial.

The link between AD and children's emotions and behavior was recognized as far back as the $1800 \mathrm{~s}$, when Wilson's Textbook of Dermatology "noted the influence on the conditions then called 'eczematous dermatoses' of 'mental emotions especially of a depressive kind'." ${ }^{24}$ In more recent years, researchers have documented evidence pointing to an increased risk of emotional and behavioral problems in children with $\mathrm{AD}$, using quantitative and qualitative study designs and employing both independent observation and parent report of child behavior and disease severity. ${ }^{6,25}$

Mothers frequently describe their infants and children with AD as being irritable and "difficult to deal with," requiring extraordinary amounts of parental attention compared with unaffected children. ${ }^{26}$ Severe pruritus contributes to irritability and lack of concentration in young children, and the need to keep the child entertained and distract them from scratching can be a major challenge for parents. ${ }^{26}$ Mothers tend to judge their infants' emotional behavior as less positive and more negative when compared with mothers of healthy control group infants, with a greater propensity to display distress when faced with limitations or sudden and novel stimuli. ${ }^{27}$

A study by Daud et $\mathrm{al}^{6}$ found that mothers more often viewed their preschool-aged children with $\mathrm{AD}$ as "poorly adaptable" with "generally difficult" behavior compared with children without AD. Disrupted sleep, clinginess, anxiety, and attention seeking were all reported to be more common among affected children, and clinical assessment identified a higher incidence of emotional and behavioral problems among children with $\mathrm{AD}$ (23\%) when compared with healthy controls (5\%). Importantly, behavioral problems appeared to be strongly associated with AD severity, ${ }^{6}$ with behavioral difficulties intensifying as skin disease worsened.

Within the pediatric $\mathrm{AD}$ literature, research examining the behavior of infants and young children has tended to describe difficulties broadly and in general terms. In contrast, research with older children has frequently classified behaviors as primarily "internalizing" (eg, fearfulness, sadness, social withdrawal) or "externalizing" (eg, disruptive, uncooperative, or aggressive behavior). In a study of children with AD aged 5-15 years, 50\% scored in the clinical range for psychological disturbance when using the Rutter A2 scale; ${ }^{28}$ however, a greater proportion of children with severe AD (80\%) scored in the clinical range compared with those with moderate $(53 \%)$ or mild (30\%) AD, or the control group $(27 \%),{ }^{7}$ again supporting the observation that more severe skin disease was linked to a more severe mental health difficulties. Along the same lines, this study reported an increased prevalence of emotional problems (40\%) and conduct disorders (10\%) among children with $\mathrm{AD}$, and overall prevalence of psychological difficulties was greater than that reported in research with children with leukemia or epilepsy. ${ }^{7}$ Other studies using objective clinician-rated assessments of $\mathrm{AD}$ severity have confirmed elevated rates of externalizing (15.6\%-17.2\%) and internalizing (25\%) child behavioral difficulties, with higher scores for behavioral difficulties once again correlating with more severe AD. ${ }^{29}$

The majority of these studies employed relatively small samples of children; however, large population-level studies have also reported similar relationships. A cross-sectional study analyzing data from 92,642 US children collected 
during the 2007 National Survey of Children's Health reported that children with $\mathrm{AD}$ had increased odds of having medically diagnosed attention-deficit hyperactivity disorder (ADHD; OR = 1.87), depression $(\mathrm{OR}=1.81)$, anxiety $(\mathrm{OR}=$ 1.77), conduct disorder $(\mathrm{OR}=1.87)$, or autism $(\mathrm{OR}=3.04) .{ }^{8}$ Notably, the increased likelihood of being diagnosed with any of these conditions strongly correlated with parent-reported AD severity, and the odds of mental health comorbidities were, by far, the highest for children with severe AD. ${ }^{8}$ The findings of this study were congruent with those of previous systematic reviews and other large-scale population surveys that reported increased risk of attention-deficit disorder (ADD)/ADHD, depression, anxiety, and conduct disorder for children with AD. ${ }^{9-12}$

Data suggest that it is not only the severity of the child's $\mathrm{AD}$ that is important in predicting likelihood of psychological comorbidity but also the number of concurrent atopic conditions a child has. A large Danish study of 9,215 children, ages 3-15 years, identified higher scores for emotional, conduct, and hyperactivity problems for children with $\mathrm{AD}$ compared with children without any atopic conditions, with odds of scoring in the clinical range on these measures increasing with the number of atopic comorbidities the child had (ie, $\mathrm{AD}$, plus asthma and/or allergic rhinitis). ${ }^{13}$ Thus, it seems that it is not only the severity of the child's skin disease but also, potentially, the combined effect of multiple chronic health problems that may be associated with psychopathology in this clinical group.

The vast majority of available research relies primarily on parent reports of child behavior and quality of life and is, therefore, subject to the limitations inherent in parent-report assessments. Nevertheless, research employing teacher-report and direct observation of child behavior has also revealed relationships congruent with the broader literature linking AD to increased internalizing and externalizing behaviors. A UK study employed teacher-reports of child behavior and child-reports of school experiences, ${ }^{14}$ revealing increased internalizing and externalizing behaviors for children with AD symptoms compared with unaffected children, as well as an increased risk of child-reported at-school bullying, thus highlighting that the impact of skin disease can affect both the home and the school environment. ${ }^{14}$ Likewise, direct observations of child and parent behavior by researchers during at-home routine skincare sessions have surprisingly strong correlations between objectively observed negative child behavior and parent-reported child behavioral difficulties $(r=0.50)$, clinician-rated AD severity $(r=0.50)$, and parent stress $(r=0.57-0.65)$, suggesting that parent-report of child behavior in this clinical group may be a more reliable form of behavioral assessment than previously thought. ${ }^{25}$

Interestingly, the link between $\mathrm{AD}$ and the presence of emotional and behavioral problems appears to persist even when the child's AD goes into remission. The German Infant Nutrition Intervention plus longitudinal cohort study revealed that children with any past history of $\mathrm{AD}$ were at increased risk of experiencing ongoing emotional, conduct, and overall behavioral difficulties at 10 years of age, even if their AD had resolved during infancy or early childhood. ${ }^{30}$ These results were supported by a second prospective population-based cohort study that identified poorer psychological well-being (externalizing behaviors) at age 8, even when children's AD symptoms were limited to infancy or the preschool years. ${ }^{15}$

The mechanisms underlying the association between AD and psychopathology in children are unknown as yet; however, authors have proposed several hypotheses that seek to explain the link between emotional and behavioral problems and $\mathrm{AD}$ in childhood. Among these is the hypothesis that higher levels of perceptual sensitivity - which appears to be more common among infants and toddlers with $\mathrm{AD}^{31,32}$ and seems to affect most modalities, but especially the tactile modality - may contribute to irritability and other behavioral problems. ${ }^{33}$ Similarly, evidence from longitudinal studies implicate $\mathrm{AD}$ and other atopic conditions in terms of disrupting normal neurodevelopment in infants ${ }^{34}$ - highlighting the potential for allergic conditions to have an additive effect in terms of risk for development of internalizing behavioral difficulties, which increases with the number of comorbid atopic conditions. ${ }^{35}$ Along similar lines, researchers have raised the possibility that the chronic inflammation associated with $\mathrm{AD}$ and other atopic conditions could be responsible, at least in part, for the increase in mental health difficulties among children with AD, particularly in light of the associations between increased levels of proinflammatory cytokines and depression, anxiety, and autism spectrum disorders in adult populations. $^{8}$

Predominantly, however, a substantial body of research implicates sleep disturbance and chronic pruritus as major contributors to difficulties of behavior and functioning for affected children. Itch and sleep disturbance are significant problems for children with AD, having a substantial impact on children's quality of life and presenting a serious and ongoing challenge to parents. ${ }^{7,26,36,37}$ Children with AD score higher than controls for sleep disturbance - particularly for difficulties with initiating and maintaining sleep and daytime sleepiness. ${ }^{38}$ Studies of preschool- and school-aged children report correlations between disrupted sleep, behavioral 
problems, and AD severity, ${ }^{38-40}$ and data suggest that sleep disruption - at least partially - mediates the relationship between behavioral difficulties and $\mathrm{AD} .{ }^{38}$ Moreover, there is some evidence to support the notion that the apparent association between AD and ADHD may be moderated by the presence of sleep difficulties; although the relationship between $\mathrm{AD}$ and ADHD appears strongest among children with reported sleep disruption, it has been found to weaken ${ }^{8}$ or become nonsignificant ${ }^{41}$ for those children without reported sleep loss. Some have even suggested that irritability and lack of concentration secondary to pruritus and scratching behaviors might mimic the phenomenology of ADHD, ${ }^{40}$ making interpretations of studies that rely on parent-report of ADHD symptoms difficult. To further complicate the issue, although there is a clear relationship between disturbed sleep and nocturnal pruritus and scratching, ${ }^{42}$ evidence suggests that disrupted sleep may even persist when AD is well controlled and symptoms are minimal. ${ }^{43}$ It is, therefore, possible that some sleep disturbance in children with $\mathrm{AD}$ may persist for behavioral reasons as opposed to being directly caused by nocturnal pruritus or scratching.

Despite ongoing conjecture over the causal mechanisms underlying these relationships, on the balance of available evidence, it can be concluded that children with AD are at increased risk of internalizing and externalizing behavioral difficulties, which can affect functioning across multiple domains and seem to be associated with more severe disease. It is notable, therefore, that greater general behavioral and emotional difficulties are also associated with increased illness-specific child behavioral difficulties such as disruptive and uncooperative behavior during $\mathrm{AD}$ treatment sessions, ${ }^{44}$ which has substantial implications for management that are discussed later.

\section{Psoriasis}

Psoriasis is a chronic, relapsing inflammatory skin condition that is characterized by the development of thickened, red, scaly lesions that may appear on any part of the body, and frequently accompanied by pain and pruritus. Generalized plaque psoriasis is the most common manifestation in children. ${ }^{45,46}$ Psoriasis has been estimated to affect $~ 2.5 \%$ of the general population, with around half of all cases diagnosed in the first 18 years of life, ${ }^{47}$ although the incidence and prevalence varies substantially with geographical region. ${ }^{48}$

Psoriasis can affect the skin, nails, and joints in both adults and children, and children may develop juvenile psoriatic arthritis as a manifestation of their psoriasis, although this is often preceded by the development of skin disease. ${ }^{49}$ Furthermore, children with psoriasis are at increased risk of developing hyperlipidemia, obesity, hypertension, diabetes mellitus, rheumatoid arthritis, and Crohn's disease, as well as mental health problems. ${ }^{47,50}$ Risk factors for the development of psoriasis include family history of the disease, which is a stronger predictor of onset in childhood compared to in adulthood. ${ }^{51}$ Importantly, onset in childhood or adolescence is associated with more severe disease and greater psychological morbidity in later life that is independent of the disease duration..$^{52}$

Although the vast majority of the literature examining relationships between psoriasis and psychological factors focuses on the adult context, the limited research available suggests that pediatric psoriasis can have a profound impact on the psychological health and quality of life of affected children. Children with psoriasis demonstrate impaired physical, emotional, social, and school functioning compared to healthy children, with impairments in quality of life that are comparable to those seen in children with other chronic diseases such as diabetes, arthritis, or asthma. ${ }^{53} \mathrm{~A}$ Swedish study reported that, for young children (ages 2-4), mood was among the most severely affected of the domains on a parent-reported child quality-of-life measure; likewise, older children (ages 5-16) report that feeling upset, sad, embarrassed, or self-conscious because of their skin is one of the most problematic issues for them. ${ }^{54}$ Poorer quality of life for children overall seems to be associated with more severe disease, ${ }^{55}$ and children report experiencing stigmatization associated with bullying, name-calling, and social rejection, and shame about the appearance of their skin, ${ }^{56}$ which can persist or worsen as they move into adolescence and adulthood..$^{57}$

To date, studies comparing psychological and mental health characteristics of children with psoriasis and healthy control children are scarce, and mostly use cross-sectional designs and small sample sizes; however, the available research suggests that children living with psoriasis are at greater risk of a range of mental health problems compared with healthy peers. A retrospective chart review of 61 pediatric psoriasis patients identified concurrent emotional stress and psychiatric morbidity in $54 \%$ and $9.8 \%$ of children, respectively. ${ }^{16}$ Another small study of 22 clinic-recruited children (ages 8-12) with psoriasis found that affected children had poorer scores on measures of depression and parent-reported health-related quality of life (psychosocial and total scores) compared with healthy controls, and that increasing disease severity was associated with worse parentreported physical health-related quality of life. ${ }^{17}$ 
Notably, the results of these smaller studies are supported by a large, retrospective, matched cohort study analyzing health data of 7,404 children with psoriasis drawn from a large US medical claims database ${ }^{18}$ that found children with psoriasis were at higher risk of being diagnosed with a mental health disorder compared with unaffected children (HR = 1.25), even after controlling for age, sex, and comorbidities, with increased risks highest for anxiety $(\mathrm{HR}=1.32)$ followed by depression ( $\mathrm{HR}=1.25$ ). In addition, they were more likely to be prescribed one or more psychotropic medications $(\mathrm{HR}=1.47)$, particularly anxiolytics $(\mathrm{HR}=2.44)$ and antidepressants $(\mathrm{HR}=1.19) .{ }^{18}$ Of particular interest with regard to the results of this study is that the rate of documented diagnosis of a mental health condition for children with psoriasis was markedly lower than the rate of psychotropic mediation use, which is suggestive of underreporting and, therefore, underestimation of the true prevalence of mental health conditions among children with psoriasis.

Compared with the body of work examining the links between $\mathrm{AD}$ and psychological well-being in children, there is limited literature examining comparable relationships in the context of pediatric psoriasis. Adult patients - many of whom experience onset of psoriasis in childhood - describe their symptoms of anxiety or depression as stemming from experiences of embarrassment, shame, or the socially limiting effects of behavior avoidance, which have been suggested to be more prominent contributors to the emotional distress experienced by individuals with psoriasis as opposed to psychological conditions from the Diagnostic and Statistical Manual of Mental Disorders. ${ }^{57}$ When examining these relationships in the reverse direction, however, numerous factors have been implicated in the onset and exacerbation of psoriasis. Stress is a well-acknowledged trigger for the onset and exacerbation of this condition, and those who experienced onset of psoriasis symptoms in childhood (rather than adulthood) seem to have an even greater susceptibility to exacerbations triggered by precipitating factors such as stress and trauma. ${ }^{51,58}$

Current perspectives favor the hypothesis that stress reactions in individuals with psoriasis are mediated by the hypothalamic-pituitary-adrenal relationship with immunologic processes.$^{58}$ Although nearly all of the available research focuses on the adult context, data suggest that up to $90 \%$ of children with psoriasis may experience stress-related exacerbations of their condition. ${ }^{59}$ This is thought to trigger a vicious cycle whereby the psoriasis acts as a source of stress for the individual, particularly when disease is severe or highly visible, and the stress that results from their disease provokes a further increase in severity of psoriasis symptoms, and so on..$^{58}$

As with $\mathrm{AD}$, the underlying cause of the apparent relationship between mental health and psoriasis in children is yet to be elucidated. However, the prevailing approach to management of psoriasis in children recognizes the profound impact on children's psychological health and quality of life; ${ }^{49}$ the importance of early diagnosis and treatment in attenuating the physical and psychological impact of the condition; ${ }^{45}$ and that psychological factors - including perceived health, perceptions of stigmatization, and depression - may be stronger determinants of disability than are disease severity, location, or duration, which highlights the importance of addressing psychological comorbidity in this clinical group. ${ }^{58,60}$

\section{Chronic urticaria}

Chronic urticaria is an inflammatory skin condition characterized by the daily or almost-daily occurrence of recurrent, transitory, intensely itchy wheals, angioedema, or both, for longer than 6 weeks. ${ }^{61}$ Compared to acute urticaria, which primarily arises from allergic or pseudoallergic reactions, the chronic form of the disease has a more complex etiology and course, and the recurrent nature and severe itch associated with the condition can be distressing and have a severe impact on quality of life for affected individuals. ${ }^{62}$

Compared to other dermatological conditions of childhood, there is a dearth of research examining chronic urticaria in the pediatric context. Until recently, there were no reliable epidemiological studies assessing the prevalence of chronic urticaria among children; however, recent estimates from a population-based Korean study of 4,079 children reported $1.8 \%$ of children affected by this condition. ${ }^{63}$ Given that the condition is relatively common among children, it is remarkable that there are so few studies examining the impact of chronic urticaria in the pediatric context, with the majority of research focused on the adult group. However, the available research suggests that urticaria has a substantial impact on children's health-related quality of life that is comparable to that of AD or psoriasis. ${ }^{4}$ A small case-control study ${ }^{19}$ identified more than double the rate of psychiatric diagnoses $(70 \%$ vs $30 \%)$ among 27 children with chronic idiopathic urticaria compared to age- and sex-matched controls. The most common diagnoses were social anxiety disorder, separation anxiety disorder, and social phobia, and scores for depression, anxiety, and internalizing problems were also higher in the chronic idiopathic urticaria group. Two thirds of children were reported to have 
experienced a stressful life event in the 6 months prior to onset of symptoms; however, there appeared to be no relationship between duration of illness and mental health. ${ }^{19}$

To date, our understanding of the links betwseen chronic urticaria and children's psychological health is constrained by a lack of research in the pediatric context. However, results from the small amount of available research are congruent with data from a comprehensive systematic review and meta-analysis of research in the adult context reporting that, compared to healthy controls, adults with chronic urticaria score higher on measures across a range of measures of psychological health including depression, anxiety, insomnia, and stressful life events. ${ }^{64}$ Moreover, research with adult patients suggests that impairments in quality of life, particularly across the domains of functioning and emotions, are most closely linked with measures of psychiatric morbidity rather than other factors such as the presence of angioedema or illness duration. ${ }^{62}$ However, the question of whether psychopathology is a consequence of chronic urticaria or a predisposing factor as yet remains unanswered. There are a number of plausible biological mechanisms by which interactions between nervous and immune systems may elicit cutaneous symptoms, and it has been proposed that stress-related or neuroendocrine mechanisms may be responsible for the link between psychological factors and the development and/or exacerbation of chronic urticaria. ${ }^{64}$

Regardless of whether psychological factors are the causes or consequences of this condition among children, there is general consensus that psychological factors play an important role in disease course and treatment efficacy, and should be included in the assessment, treatment, and monitoring phases of medical management. ${ }^{19,62}$

\section{Implications for management}

The importance of taking into account the relatively high rate of emotional and behavioral difficulties in the management of children with dermatological conditions is twofold. First, the balance of evidence suggests that improving disease control and reducing symptom severity may help alleviate at least some of children's emotional and behavioral difficulties. Second, fewer emotional and behavioral difficulties may reduce triggers for exacerbations of dermatological symptoms and also facilitate more consistent and effective disease management - ultimately reducing disease severity.

In the pediatric dermatology context, involvement and commitment from parents and caregivers are crucial to the successful management of the child's condition; ${ }^{65}$ it is, therefore, important that parents are not only educated about the child's disease and treatment but have the skills and confidence necessary to be able to implement the child's treatment plan consistently and effectively. ${ }^{66}$ Adherence to treatment in dermatology, in general, and pediatric dermatology, in particular, is notoriously low, ${ }^{67,68}$ leading to increased morbidity and health care utilization. ${ }^{69}$ This is perhaps unsurprising, given the complex routines of trigger avoidance and skincare treatment that are frequently needed to control symptoms and reduce flares, which can be time consuming and unpleasant for both the child and the parent. ${ }^{26,70}$

The presence of concurrent child behavioral or emotional problems can make implementing treatment recommendations difficult for parents, undermining parents' confidence with performing management tasks, and impeding treatment and longer-term management. Parents report children's lack of cooperation with treatment as a common problem, ${ }^{71}$ and dealing with difficult child behaviors during skincare sessions can be challenging for parents. ${ }^{44}$ General child behavioral difficulties as well as those specific to the child's illness or their treatment are important predictors of parents' self-efficacy with managing their child's condition and success with performing illness management tasks. ${ }^{29,44}$ Moreover, parents' use of ineffective parenting strategies (eg, laxness, overreactivity) has been associated with less successful disease management and greater disease severity, and parents who report greater self-efficacy with illness management also tend to report greater self-efficacy with managing their child's behavior. ${ }^{25}$ This raises the possibility that helping parents develop the skills and confidence to manage their child's emotions and behavior as well as manage their child's illness may be a more effective way to improve illness management and child health outcomes than addressing these issues separately.

Evaluation of interventional approaches in other pediatric chronic illnesses suggests that the most successful treatment programs address multiple factors that contribute to chronic illness management and control, which includes psychosocial factors such as child emotional and behavioral difficulties and parents' or caregivers' needs for education and support. ${ }^{72}$ To date, however, systematic reviews of educational and psychological interventions have found limited evidence for the effectiveness of educational and psychological interventions in terms of improving outcomes for children with chronic dermatological conditions. ${ }^{65}$ Despite this, there is an emerging literature examining the potential of parentfocused behavioral interventions - particularly, parenting interventions - to improve outcomes for children with chronic health problems. ${ }^{66}$ Evidence suggests that parenting interventions may be an effective approach for fostering 
parenting skills and confidence with AD management, leading to reductions in child behavioral difficulties, decreases in parent-reported skin-disease severity, less use of ineffective parenting behaviors, and reduced parent stress. ${ }^{73-75}$ Likewise, resilience has been identified as an important predictor of internalizing and externalizing child behaviors, and strategies to enhance children's resilience, such as promoting parenting skills, self-efficacy, and nurturing relationships between the child and peers/family, have been suggested as important to include in holistic care plans for children with skin disease. ${ }^{76}$

Along these lines, recent approaches have sought to integrate medical and psychological health care in pediatric dermatology, and the feasibility of integrating medical and psychological care services for children and their families has been demonstrated..$^{77}$ Evidence suggests disease-related problems and distress (eg, child sleep difficulties, feeling overwhelmed, or having trouble following treatment recommendations) are stronger predictors of help-seeking by parents than the child's disease severity. ${ }^{77}$ The fact that parents not only seek help with disease management but also general behavioral and emotional concerns lends support to the notion that integrating psychological services into routine medical care may increase acceptability to parents, reduce stigma, and provide a portal for families to access other mental health services. ${ }^{77}$ Integrated care has the potential to facilitate routine screening for emotional and behavioral difficulties in children with dermatological conditions, which may be even more salient in pediatric - compared to adult - patient populations. For example, the early onset of psoriasis in childhood has been linked with greater familial susceptibility to the disease, more severe disease course, and greater impact on psychosocial functioning in later life; thus, early detection of emotional and behavioral concerns in children may be considered a clinical priority, ${ }^{52}$ enabling early intervention and reducing the impact of the illness and treatment on children's physical, emotional, and social growth and development.

\section{Patient-specific considerations and impact on quality of life}

Evidence from the broader child health literature shows that behavioral and emotional difficulties are more common among children with chronic health conditions, ${ }^{78}$ and the impact on children's general development, learning, behavior and emotions, and overall morbidity increases sharply with the number of chronic health problems a child has. ${ }^{79}$ This is particularly salient given the comorbid nature of many dermatological conditions and their likelihood of occurring in combination with chronic health problems in other systems (eg, AD with asthma and allergic rhinitis; the "allergic triad"). Thus, psychosocial assessment and care planning for children with dermatological conditions must take into account other illness diagnoses and treatment plans, to ensure that multiple risk factors - where they exist - are assessed for and addressed. Recognizing that children with multiple conditions may be at greater risk for psychological difficulties - and aiming for early assessment and intervention, where indicated - is key.

Quality-of-life measures are being targeted in the drive to establish sets of core outcome measures to be used in the evaluation of pharmacological and psychosocial treatments and interventions for individuals with dermatological conditions..$^{80}$ As previously outlined, children with chronic skin disease suffer similar impairments to their quality of life as do children with other chronic health problems, ${ }^{4}$ and the impact of the conditions and their treatment can extend beyond the child to impact the quality of life of their parents and siblings, who may experience similar challenges and restrictions (eg, disrupted sleep, restricted activities) as do affected children. ${ }^{26,37,81,82}$ Notably, children's self-rated quality of life has been demonstrated to improve following pharmacotherapy ${ }^{83,84}$ - particularly where improvements in itch and sleep disturbance are achieved ${ }^{84}$ - and improvements in quality of life correlate with improvements in disease severity following treatment. ${ }^{84}$ However, it should be noted that some impacts on quality of life may persist beyond the phase of active disease and continue even when the condition is in remission, ${ }^{43}$ which may require a more direct approach involving behavioral intervention. On the other hand, behavioral intervention approaches have proven successful in improving parent-rated quality of life for themselves and their family even when the target child's disease is still active, ${ }^{74}$ and approaches that primarily target parents' well-being and stress management may be effective in improving parent and family quality of life.

\section{Methodological challenges}

Methodological challenges have been identified by researchers attempting to disentangle the relationships between dermatological conditions and both internalizing and externalizing behavioral difficulties in children. The type of behavioral or emotional problems observed in children with dermatological conditions varies across studies, as have the sample characteristics and the measures employed. Wide variations in age and disease severity of child samples - as well as in measures of disease severity and child behavior - make comparisons 
between studies difficult. Reliance on parent-report of child behavior increases the risk of bias in most studies, although the use of teacher-report or direct observation helps circumvent this issue. Although some have used objective data to confirm dermatological and/or psychological diagnoses, methodological concerns in terms of inadequate definition of dermatological and psychological variables (eg, parentreport of symptoms) means that the results of many studies included in this review must be interpreted with caution. ${ }^{85}$

Finally, in contrast to the substantial and growing body of literature examining relationships between AD and children's psychological adjustment, there is a distinct dearth of comparable literature in the contexts of pediatric psoriasis and urticaria. A recent scoping review of the epidemiology of childhood psoriasis highlighted that the overall number of publications on childhood psoriasis has only increased notably in recent years, with $70 \%$ of studies published in the last decade. ${ }^{86}$ Commentators have observed that this increase in attention within the literature coincides with the 2008 approval of the first biologic for severe plaque psoriasis in children in Europe. ${ }^{87}$ Moreover, limitations due to study design and heterogeneity in methodology and outcomes limit the conclusions that can be drawn. Large, well-designed epidemiological studies are needed to enable researchers and clinicians to draw firm conclusions about the prevalence and correlates of emotional and behavioral difficulties among children with dermatological conditions and evaluate the efficacy of interventions designed to improve management and child health outcomes.

\section{Conclusion}

Available research suggests that children affected by three common dermatological conditions of childhood - AD, psoriasis, and urticaria - are at increased risk of behavioral difficulties, which may be associated with increased disease severity, impaired quality of life, and disease management difficulties. However, a general paucity of research combined with limitations in terms of study design, variability in operationalization of constructs, and heterogeneity in measurement of outcomes makes it difficult to draw firm conclusions in this area. Prospective, controlled studies that use consistent and well-validated methods to both define and assess skin disease as well as assess emotional and behavioral difficulties, health-related quality of life, and objective sleep disturbance are needed to better understand the relationship between dermatological and psychological conditions in children. Future research will elucidate the mechanisms underlying the relationships between dermatological and psychological health in children, which are yet unknown.

\section{Acknowledgment}

This research was supported by the Children's Hospital Foundation Early Career Fellowship (award ref. no. 50223).

\section{Disclosure}

The Parenting and Family Support Centre is partly funded by royalties stemming from published resources of the Triple $\mathrm{P}$ - Positive Parenting Program, which is developed and owned by The University of Queensland (UQ). Royalties are also distributed to the Faculty of Health and Behavioural Sciences at UQ and contributory authors of published Triple P resources. Triple P International (TPI) Pty Ltd is a private company licensed by Uniquest Pty Ltd on behalf of UQ, to publish and disseminate Triple $\mathrm{P}$ worldwide. The author of this report has no share or ownership of TPI. TPI had no involvement in the study design, collection, analysis or interpretation of data, or writing of this report. Dr Mitchell is an employee at UQ. The author reports no other conflicts of interest in this work.

\section{References}

1. Bayram C, Harrison C, Charles J, Britt H. 'The kids are alright' - changes in GP consultations with children 2000-2015. Aust Fam Physician. 2015;44(12):877-879.

2. Hay RJ, Johns NE, WIlliams HC, et al. The global burden of skin disease in 2010: an analysis of the prevalence and impact of skin conditions. J Invest Dermatol. 2014;134(6):1527-1534.

3. Brown MM, Chamlin SL, Smidt AC. Quality of life in pediatric dermatology. Dermatol Clin. 2013;31(2):211-221.

4. Beattie PE, Lewis-Jones MS. A comparative study of impairment of quality of life in children with skin disease and children with other chronic childhood diseases. Br J Dermatol. 2006;155(1):145-151.

5. Gupta MA, Gupta AK. Psychiatric and psychological co-morbidity in patients with dermatologic disorders: epidemiology and management. Am J Clin Dermatol. 2013;4(12):833-842.

6. Daud LR, Garralda ME, David TJ. Psychosocial adjustment in preschool children with atopic eczema. Arch Dis Child. 1993;69(6):670-676.

7. Absolon CM, Cottrell D, Eldridge SM, Glover MT. Psychological disturbance in atopic eczema: the extent of the problem in school-aged children. Br J Dermatol. 1997;137(2):241-245.

8. Yaghmaie P, Koudelka CW, Simpson EL. Mental health comorbidity in patients with atopic dermatitis. J Allergy Clin Immunol. 2016;131(2):428-433.

9. Garg N, Silverberg JI. Association between childhood allergic disease, psychological comorbidity, and injury requiring medical attention. Ann Allergy Asthma Immunol. 2014;112(6):525-532.

10. Liao TC, Lien YT, Wang S, Huang SL, Chen CY. Comorbidity of atopic disorders with autism spectrum disorder and attention deficit/ hyperactivity disorder. J Pediatr. 2016;171:248-255.

11. Lin YT, Chen YC, Gau SS, et al. Associations between allergic diseases and attention deficit hyperactivity/oppositional defiant disorders in children. Pediatr Res. 2016;80(4):480-485.

12. Schmitt J, Buske-Kirschbaum A, Roessner V. Is atopic disease a risk factor for attention-deficit/hyperactivity disorder? A systematic review. Allergy. 2010;65(12):1506-1524. 
13. Hammer-Helmich L, Linneberg A, Obel C, Thomsen SF, Mollehave LT, Glumer C. Mental health associations with eczema, asthma and hay fever in children: a cross-sectional study. BMJ Open. 2016;6(10):e012637.

14. Teyhan A, Galobardes B, Henderson J. Child allergic symptoms and well-being at school: findings from ALSPAC, a UK cohort study. PLoS One. 2015;10(8):e0135271.

15. Teyhan A, Galobardes B, Henderson J. Child allergic symptoms and mental well-being: the role of maternal anxiety and depression. J Pediatr. 2015;165(3):592-599.

16. Seyhan M, Coşkun BK, Sağlam H, Ozcan H, Karincaoğlu Y. Psoriasis in childhood and adolescence: evaluation of demographic and clinical features. Pediatr Int. 2006;48(6):525-530.

17. Bilgic A, Bilgic Ö, Akiş HK, Eskioğlu F, Kihç EZ. Psychiatric symptoms and health-related quality of life in children and adolescents with psoriasis. Pediatr Dermatol. 2010;27(6):614-617.

18. Kimball AB, Wu EQ, Guerin A, et al. Risks of developing psychiatric disorders in pediatric patients with psoriasis. $J$ Am Acad Dermatol. 2012;67(4):651-657.

19. Hergüner S, Kiliç G, Karakoç S, Tamay Z, Tüzün U, Güler N. Levels of depression, anxiety and behavioural problems and frequency of psychiatric disorders in children with chronic idiopathic urticaria. $\mathrm{Br}$ $J$ Dermatol. 2011;164(6):1342-1347.

20. Williams H, Stewart A, von Mutius E, Cookson W, Anderson HR; International Study of Asthma and Allergies in Childhood (ISAAC) Phase One and Three Study Groups. Is eczema really on the increase worldwide? J Allergy Clin Immunol. 2008;121(4):947-954.e15.

21. Paller AS, Mancini AJ. Eczematous eruptions in childhood. In: Paller AS, Mancini AJ, editors. Hurwitz Clinical Pediatric Dermatology. 3rd ed. Philadelphia: Elsevier Saunders; 2005:49-64.

22. Ben-Gashir MA, Seed PT, Hay RJ. Predictors of atopic dermatitis severity over time. J Am Acad Dermatol. 2004;50(3):349-356.

23. Krafchik BR, Halbert A, Yamamoto K, Sasaki R. Eczematous dermatitis. In: Schachner L, Hansen RC, editors. Pediatric Dermatology. 3rd ed. Philadelphia: Mosby; 2003:609-630.

24. Whittkowker ED, Hunt BR. Psychological aspects of atopic dermatitis in children. Can Med Assoc J. 1958;79(10):810-817.

25. Mitchell AE, Fraser JA, Morawska A, Ramsbotham J, Yates P. Parenting and childhood atopic dermatitis: a cross-sectional study of relationships between parenting behaviour, skin care management, and disease severity in young children. Int J Nurs Stud. 2016;64:72-85.

26. Elliott BE, Luker K. The experiences of mothers caring for a child with severe atopic eczema. J Clin Nurs. 1997;6(3):241-247.

27. Pauli-Pott U, Darui A, Beckmann D. Infants with atopic dermatitis: maternal hopelessness, child-rearing attitudes and perceived infant temperament. Psychother Psychosom. 1999;68(1):39-45.

28. Rutter M, Cox A, Tupling C, Berger M, Yule W. Attainment and adjustment in two geographical areas. I- the prevalence of psychiatric disorder. Br J Psychiatry. 1975;126:493-509.

29. Mitchell AE, Fraser JA, Ramsbotham J, Morawska A, Yates P. Childhood atopic dermatitis: a cross-sectional study of relationships between child and parent factors, atopic dermatitis management, and disease severity. Int J Nurs Stud. 2015;52(1):216-218.

30. Schmitt J, Apfelbacher C, Chen CM, et al; German Infant Nutrition Intervention plus Study Group. Infant-onset eczema in relation to mental health problems at age 10 years: results from a prospective birth cohort study (German Infant Nutrition Intervention plus). J Allergy Clin Immunol. 2010;125(2):404-410.

31. Pourpak Z, Sedighipour L, Firooz A, et al. Behavioral characteristics in 3- to 12-month-old infant with atopic dermatitis: a case-control study. Pediatr Allergy Immunol. 2007;18(4):339-345.

32. Sedighipour L, Firooz A, Pourpak Z, et al. Behavioural characteristics in 18- to 36-month-old toddlers with atopic dermatitis compared with healthy children. Pediatr Dermatol. 2010;27(3):314-315.

33. Engel-Yeger B, Habib-Mazawi S, Parush S, Rozenman D, Kessel A, Shani-Adir A. The sensory profile of children with atopic dermatitis as determined by the sensory profile questionnaire. $\mathrm{J} \mathrm{Am} \mathrm{Acad} \mathrm{Dermatol}$. 2007;57(4):610-615.
34. Meldrum SJ, D'Vaz N, Dunstan JA, et al. Allergic disease in the first year of life is associated with differences in subsequent neurodevelopment and behaviour. Early Hum Dev. 2012;88(7):567-573.

35. Nanda MK, LeMasters GK, Levin L, et al. Allergic diseases and internalizing behaviors in early childhood. Pediatrics. 2016;137(1):e20151922.

36. Hon KL, Leung TF, Wong KY, Chow CM, Chuh A, Ng PC. Does age or gender influence quality of life in children with atopic dermatitis? Clin Exp Dermatol. 2008;33(6):705-709.

37. Lewis-Jones S. Quality of life and childhood atopic dermatitis: the misery of living with childhood eczema. Int J Clin Pract. 2006;60(8): 984-992.

38. Camfferman D, Kennedy JD, Gold M, Martin AJ, Winwood P, Lushington K. Eczema, sleep, and behavior in children. J Clin Sleep Med. 2010;6(6):581-588.

39. Dahl RE, Bernhisel-Broadbent J, Scanlon-Holdford S, Sampson HA, Lupo M. Sleep disturbances in children with atopic dermatitis. Arch Pediatr Adolesc Med. 1995;149(8):856-860.

40. Chang HY, Seo JH, Kim HY, et al. Allergic diseases in preschoolers are associated with psychological and behavioural problems. Allergy Asthma Immunol Res. 2013;5(5):315-321.

41. Romanos M, Gerlach M, Warnke A, Schmitt J. Association of attentiondeficit/hyperactivity disorder and atopic eczema modified by sleep disturbance in a large population-based sample. J Epidemiol Community Health. 2010;64(3):269-273.

42. Stores G, Burrows A, Crawford C. Physiological sleep disturbance in children with atopic dermatitis: a case control study. Pediatr Dermatol. 1998;15(4):264-268.

43. Reuveni H, Chapnick G, Tal A, Tarasiuk A. Sleep fragmentation in children with atopic dermatitis. Arch Pediatr Adolesc Med. 1999;153(3): 249-253.

44. Mitchell AE, Morawska A, Fraser JA, Sillar K. Child behaviour problems and childhood illness: development of the Eczema Behaviour Checklist. Child Care Health Dev. 2017;43(1):67-74.

45. de Moll EH, Chang MW, Strober B. Psoriasis in adults and children: kids are not just little people. Clin Dermatol. 2016;34(6):717-723.

46. Morris A, Rogers M, Fischer G, Williams K. Childhood psoriasis: a clinical review of 1262 cases. Pediatr Dermatol. 2001;18(3):188-198.

47. Augustin M, Glaeske G, Radtke MA, Christophers E, Reich K, Schafer I. Epidemiology and comorbidity of psoriasis in children. Br J Dermatol. 2010;162(3):633-636.

48. Parisi R, Symmons DPM, Griffiths CEM, Ashcroft DM, Indentification and Management of Psoriasis and Associated ComorbidiTy (IMPACT) project team. Global epidemiology of psoriasis: a systematic review of incidence and prevalence. J Invest Dermatol. 2013;133(2):377-385.

49. Bronckers IM, Paller AS, van Geel MJ, van de Kerkhof PC, Seyger MM. Psoriasis in children and adolescents: diagnosis, management and comorbidities. Pediatr Drugs. 2015;17(5):373-384.

50. Matusiewicz D, Koerber A, Schadendorf D, Wasem J, Neumann A. Childhood psoriasis - an analysis of German health insurance data Pediatr Dermatol. 2014;31(1):8-13.

51. Raychaudhuri SP, Gross J. A comparative study of pediatric onset psoriasis with adult onset psoriasis. Pediatr Dermatol. 2000;17(3):174-178.

52. Remröd C, Sjöström K, Svensson A. Psychological differences between early- and late-onset psoriasis: a study of personality traits, anxiety and depression in psoriasis. Br J Dermatol. 2013;169(2):344-350.

53. Varni JW, Globe DR, Gandra SR, Harrison DJ, Hooper M, Baumgartner S. Health-related quality of life of pediatric patients with moderate to severe plaque psoriasis: comparisons to four common chronic diseases. Eur J Pediatr. 2012;171(3):485-492.

54. Gånemo A, Wahlgren CF, Svensson A. Quality of life and clinical features in Swedish children with psoriasis. Pediatr Dermatol. 2011;28(4):375-379.

55. de Jager ME, van de Kerkhof PC, de Jong EM, Seyger MM. A crosssectional study using the Children's Dermatology Life Quality Index (CDLQI) in childhood psoriasis: negative effect on quality of life and moderate correlation of CDLQI with severity scores. Br J Dermatol. 2010;163(5):1099-1101. 
56. de Jager ME, de Jong EM, Evers AW, van de Kerkhof PC, Seyger MM. The burden of childhood psoriasis. Pediatr Dermatol. 2011;28(6):736.

57. Magin P, Adams J, Heading G, Pond D, Smith W. The psychological sequelae of psoriasis: results of a qualitative study. Psychology Health Med. 2009;14(2):150-161.

58. Basavaraj KH, Navya MA, Rashmi R. Stress and quality of life in psoriasis: an update. Int J Dermatol. 2011;50(7):783-792.

59. Nyfors A, Lemholt K. Psoriasis in children. A short review and a survey of 245 cases. Br J Dermatol. 1975;92(4):437-442.

60. Richards HL, Fortune DG, Griffiths CE, Main CJ. The contribution of perceptions of stigmatisation to disability in patients with psoriasis. J Psychosom Res. 2001;50(1):11-15.

61. Zuberbier T, Asero R, Bindslev-Jensen C, et al; Dermatology Section of the European Academy of Allergology and Clinical Immunology; Global Allergy and Asthma European Network; European Dermatology Forum; World Allergy Organization. EAACI/GA²LEN/EDF/WAO guildeline: definition, classification and diagnosis of urticaria. Allergy. 2009;64(10): 1417-1426.

62. Staubach P, Eckhardt-Henn A, Dechene M, et al. Quality of life in patients with chronic urticaria is differentially impaired and determined by psychiatric comorbidity. Br J Dermatol. 2006;154(2):294-298.

63. Lee SJ, Ha EK, Jee HM, et al. Prevalence and risk factors of urticaria with a focus on chronic urticaria in children. Allergy Asthma Immunol Res. 2017;9(3):212-219.

64. Ben-Shoshan M, Blinderman I, Raz A. Psychological factors and chronic spontaneous urticaria: a systematic review. Allergy. 2013;68(2):131-141.

65. Ersser SJ, Cowdell F, Latter S, et al. Psychological and educational interventions for atopic eczema in children. Cochrane Database Syst Rev. 2014;(1):CD004054.

66. Morawska A, Fraser J, Calam R. Parenting interventions for childhood chronic illness: a review and recommendations for intervention delivery and design. $J$ Child Health Care. 2015;19(1):5-17.

67. Krejci-Manwaring J, Tusa MG, Carroll C, et al. Stealth monitoring of adherence to topical medication: adherence is very poor in children with atopic dermatitis. J Am Acad Dermatol. 2007;56(2):211-216.

68. Augustin M, Holland B, Dartsch D, Langenbruch A, Radtke MA. Adherence in the treatment of psoriasis: a systematic review. Dermatology. 2011;222(4):363-374.

69. Bass AM, Anderson KL, Feldman SR. Interventions to increase treatment adherence in pediatric atopic dermatitis: a systematic review. J Clin Med. 2015;4(2):231-242.

70. Chamlin SL, Frieden IJ, Williams ML, Chren MM. Effects of atopic dermatitis on young American children and their families. Pediatrics. 2004;114(3):607-611.

71. Fischer G. Compliance problems in paediatric atopic eczema. Australas J Dermatol. 1996;37(Suppl 1):s10-s13.

72. Clark NM, Mitchell HE, Rand CS. Effectiveness of educational and behavioral asthma interventions. Pediatrics. 2009;123(S3):S185-S192.
73. Morawska A, Mitchell AE, Burgess S, Fraser J. Randomized controlled trial of Triple $\mathrm{P}$ for parents of children with asthma or eczema: effects on parenting and child behavior. J Consult Clin Psychol. 2017;85(4):283-296.

74. Morawska A, Mitchell AE, Burgess S, Fraser J. Effects of Triple P parenting intervention on child health outcomes for childhood asthma and eczema: randomised controlled trial. Behav Res Ther. 2016;83: $35-44$.

75. Morawska A, Mitchell AE, Burgess S, Fraser J. Fathers' perceptions of change following parenting intervention: randomized controlled trial of Triple $\mathrm{P}$ for parents of children with asthma or eczema. $J$ Pediatr Psychol. 2017;42(7):792-803.

76. Kim DH, Im YJ. Resilience as a protective factor for the behavioral problems in school-aged children with atopic dermatitis. J Child Health Care. 2014;18(1):47-56.

77. LeBovidge JS, Kelley SD, Lauretti A, et al. Integrating medical and psychological health care for children with atopic dermatitis. J Pediatr Psychol. 2007;32(5):617-625.

78. Pinquart M, Shen Y. Behavior problems in children and adolescents with chronic physical illness: a meta-analysis. $J$ Pediatr Psychol. 2011;36(9):1003-1016.

79. Newacheck PW, Stoddard JJ. Prevalence and impact of mulitple childhood chronic illnesses. J Pediatr. 1994;124(1):40-48.

80. Schmitt J, Apfelbacher C, Spuls PI, et al. The Harmonizing Outcome Measures for Eczema (HOME) roadmap: a methodological framework to develop core sets of outcome measures in dermatology. J Invest Dermatol. 2015;135(1):24-30.

81. Faught J, Bierl C, Barton B, Kemp A. Stress in mothers of young children with eczema. Arch Dis Child. 2007;92(8):683-686.

82. Lawson V, Lewis-Jones MS, Finlay AY, Reid P, Owens RG. The family impact of childhood atopic dermatitis: the Dermatitis Family Impact questionnaire. Br J Dermatol. 1998;138(1):107-113.

83. Langley RG, Paller AS, Herbert AA, et al. Patient-reported outcomes in pediatric patients with psoriasis undergoing etanercept treatment: 12-week results from a phase III randomized controlled trial. JAm Acad Dermatol. 2010;64(1):64-70.

84. Oostveen AM, de Jager ME, van de Kerkhof PC, Donders AR, de Jong EM, Seyger MM. The influence of treatments in daily clinical practice on the Children's Dermatology Life Quality Index in juvenile psoriasis: a longitudinal study from the Child-CAPTURE patient registry. $\mathrm{Br} \mathrm{J}$ Dermatol. 2012;167(1):145-149.

85. Gee SN, Bigby M. Atopic dermatitis and attention-deficit/hyperactivity disorder: is there an association? Allergy. 2014;65(12):1506-1524.

86. Burden-Teh E, Thomas KS, Ratib S, Grindlay D, Adaji E, Murphy R. The epidemiology of childhood psoriasis: a scoping review. Br J Dermatol. 2016;174(6):1242-1257.

87. Dowlatshahi EA, Wakkee M. Rising interest in the field of paediatric psoriasis. Br J Dermatol. 2016;174(6):1242-1257.
Psychology Research and Behavior Management

\section{Publish your work in this journal}

Psychology Research and Behavior Management is an international, peerreviewed, open access journal focusing on the science of psychology and its application in behavior management to develop improved outcomes in the clinical, educational, sports and business arenas. Specific topics covered in the journal include: Neuroscience, memory and decision making; Behavior

\section{Dovepress}

modification and management; Clinical applications; Business and sports performance management; Social and developmental studies; Animal studies. The manuscript management system is completely online and includes a very quick and fair peer-review system, which is all easy to use. Visit http://www. dovepress.com/testimonials.php to read real quotes from published authors. 